Canad. Math. Bull. Vol. 63 (4), 2020 pp. 891-900

http://dx.doi.org/10.4153/S0008439520000120

(c) Canadian Mathematical Society 2020 This is an Open Access article, distributed under

the terms of the Creative Commons Attribution licence (http://creativecommons.org/licenses/by/4.0/), which permits unrestricted re-use, distribution, and reproduction in any medium, provided the original work is properly cited.

\title{
SNC Log Symplectic Structures on Fano Products
}

\author{
Katsuhiko Okumura
}

Abstract. This paper classifies Poisson structures with the reduced simple normal crossing divisor on a product of Fano varieties of Picard number 1 . The characterization of even-dimensional projective spaces from the viewpoint of Poisson structures is given by Lima and Pereira. In this paper, we generalize the characterization of projective spaces to any dimension.

\section{Introduction}

Geometry of log symplectic form is well investigated, and the term log symplectic is slightly abused. In the field of differential geometry, it mostly refers to a generically symplectic Poisson structure with the reduced and smooth divisor $[1,5]$. Such a structure is often called topologically stable Poisson [16], b-Poisson [7], or b-log symplectic [11] in the field of topological geometry. In Goto's definition [4], log symplectic indicates the generically symplectic Poisson strucuture with the reduced and simple normal crossing degeneracy divisor. In this paper, we use this terminology following Pym's definition [14]. That is, we do not suppose that the reduced degeneracy divisor is smooth or simple normal crossing. This is a reasonable definition in terms of holomorphic Poisson structures and the field of algebraic geometry, as the degeneracy divisors usually have singularities in the higher-dimensional case [6, Theorem 23]. In fact, some of the Feigin and Odesskii's examples have elliptic singular points.

One of the main benefits of holomorphic Poisson structure is that holomorphic Poisson manifolds also have the almost complex structure. Interestingly, in terms of the symplectic geometry, the known examples of projective irreducible holomorphic symplectic manifolds are only of four types [12]. Therefore, the analogous problem is worth investigating. Since Poisson structures can be regarded as a generalization of symplectic forms, there should be many more types of such Poisson manifolds. Indeed, one can easily find such examples on the projective space. However, the following theorem suggests that the Poisson structure still imposes severe constraints.

Theorem $1.1([10]) \quad$ Let $(X, \Pi)$ be a log symplectic structure with the simple normal crossing degeneracy divisor (say snc log symplectic structure) on a complex Fano variety $X$ with cyclic Picard group of even dimension $2 n \geq 4$. Then $X$ is a projective space, and $\Pi$ is a diagonal Poisson structure.

Received by the editors June 18, 2019; revised September 9, 2019.

Published online on Cambridge Core February 24, 2020.

AMS subject classification: 53D17, $14 \mathrm{~J} 45$.

Keywords: Poisson structure, symplectic, projective space. 
The diagonal Poisson structure on a projective space (or an affine space) is defined as a generically symplectic Poisson structure whose degeneracy divisor is the union of all coordinate hyperplanes. A local form of a diagonal Poisson structure can be given by $\Pi=\sum_{i<j} c_{i j} x_{i} x_{j} \frac{\partial}{\partial x_{i}} \wedge \frac{\partial}{\partial x_{j}}$ for a general system of coefficients $c_{i j}$ and homogeneous coordinates $x_{i}$ of $X$.

The degeneracy divisor must have singularities when we treat the higherdimensional variety, so we usually assume a fixed kind of the singularity of the degeneracy divisor. This principle is justified, because the Zariski closure of set of $\log$ symplectic structures or the set of SNC log symplectic structures forms a union of connected components in the moduli space of all Poisson bivector fields [15].

There are two cases used to classify holomorphic log symplectic manifolds. One is when the singularity is not a simple normal crossing singularity, and the other is when the Picard group of a variety is not cyclic. Pym [15] researches the case where all the singular points are elliptic singular points. So one of the main unsolved situations is that the variety is not of Picard rank 1. The author [13] classifies the case of the blowing up of projective spaces along a linear subspace. The focus of this paper is a product of varieties.

In this paper, we prove the following theorem.

Theorem 1.2 Let $X_{i}$ be a complex Fano variety of Picard number 1 and of dimension $n_{i} \geq 3$, let $X=\prod_{i=1}^{m} X_{i}$ be a product of even dimension $2 n=\sum_{i=1}^{m} n_{i}$, and let $\Pi$ be a SNC log symplectic structure on $X$. Then we have $X_{i}=\mathbb{P}^{n_{i}}$, and $\Pi$ is a diagonal Poisson structure.

A diagonal Poisson structure on the product of projective spaces indicates that the degeneracy divisor is the union of all coordinate hyperplanes. Let $x_{i 1}, \ldots, x_{i n_{i}}$ denote a homogeneous coordinate system of $X_{i}$ and $c_{i j k l} \in \mathbb{C}$. Then we can express the diagonal Poisson structure on the product of the projective spaces in the following form:

$$
\Pi=\sum_{\substack{1 \leq i, k \leq m \\ 0 \leq j \leq n_{i} \\ 0 \leq l \leq n_{k}}} c_{i j k l} x_{i j} x_{k l} \frac{\partial}{\partial x_{i j}} \wedge \frac{\partial}{\partial x_{k l}} .
$$

This theorem gives the characterization of projective spaces of any dimensions.

Corollary 1.3 Let $X$ be a complex Fano variety of Picard number 1 . Suppose that $X \times X$ admits a SNC log symplectic structure, then $X$ is a projective space.

\section{Poisson Structures}

Only in this chapter, we assume that the base field is an algebraivally closed and of characteristic 0 . Let $X$ be a smooth projective variety. A Poisson structure on $X$ is a bivector field $\Pi \in \Gamma\left(X, \wedge{ }^{2} \mathcal{T}_{X}\right)$ such that the Schouten bracket $[\Pi, \Pi] \in \Gamma\left(X, \wedge{ }^{3} \mathcal{T}_{X}\right)$ vanishes identically. When we define a bilinear map by $\{f, g\}=\Pi(d f, d g)$ for $f$, $g \in \mathcal{O}_{X}$, the bracket satisfies the following properties: 
(a) (skew-symmetric) $\{f, g\}=-\{g, f\}$,

(b) (Jacobi identity) $\{f,\{g, h\}\}+\{g,\{h, f\}\}+\{h,\{f, g\}\}=0$,

(c) (Leibniz rule) $\{f, g \cdot h\}=\{f, g\} h+g\{f, h\}$.

In general, (a) and (c) hold for every bracket defined by a bivector field. Jacobi identity holds if and only if the bivector fields vanishes the Schouten bracket.

We say a Poisson structure $\Pi$ has rank $2 k$ at a point $x \in X$ if $\Pi^{k}(x) \neq 0$ and $\Pi^{k+1}(x)=0$. For the largest number $k$ that satisfies $\Pi^{k} \neq 0$, we say $\Pi$ is of rank $2 k$. If $\operatorname{dim} X=2 n$ and $\operatorname{rank} \Pi=2 n$, then we call the Poisson structure $\Pi$ generically symplectic. We set $D_{2 k-2}(\Pi):=\left\{x \in X \mid \operatorname{rank}_{x} \Pi<2 k\right\}$. We call this set $2 k$-th degeneracy locus. If $\Pi$ has rank $2 k$, then the divisorial part of $D_{2 k-2}(\Pi)$ is called the degeneracy divisor of $\Pi$, and we denote it by $D(\Pi)$. If $\Pi$ is a generically symplectic Poisson structure, then the degeneracy loci forms a divisor, that is, $D(\Pi)=D_{2 n-2}(\Pi)=$ $\left\{x \in X \mid \Pi^{n}(x)=0\right\}$. For a projective variety $X$, as $\Pi^{n}$ is a holomorphic section of $\wedge^{2 n} \mathcal{T}_{X}=-\mathcal{K}_{X}, D(\Pi)$ is an effective anti-canonical divisor.

\section{Outline of Pym's Proof}

In this section, we review an outline of the proof of Theorem 1.1 given by Pym. For the product case, the proof will also be given along this outline. Thereafter, we assume that a variety $X$ is over a complex number field $\mathbb{C}$. Pym's proof is consist of three steps. First claim is that Fano index of $X$ has inequality

$$
i_{X} \geq 2 n-1 \text {. }
$$

This claim can be proved by an inductive argument on the dimension of $\mathrm{X}$. The key ideas of the induction are generalized in Lemmas 5.1 and 5.2 in this paper. The classification of Fano varieties with high-index are well known.

Theorem $3.1([2,3,8]) \quad$ Let $X$ be a $n$-dimensional Fano variety of Picard number 1. Suppose $i_{X} \geq n-1$. Then $X$ is in one of the following cases:

(i) $i_{X}=n+1$ and $X=\mathbb{P}^{n}$;

(ii) $i_{X}=n$ and $X=Q^{n} \subset \mathbb{P}^{n+1}$, a smooth quadric hypersurface;

(iii) $i_{X}=n-1$ and $X$ is one of the following varieties:

(a) a degree-six hypersurface in the $(n+1)$-dimensional weighted projective space $\mathbb{P}(3,2,1, \ldots, 1)$;

(b) a double cover of $\mathbb{P}^{n}$ branched over a smooth quartic hypersurface;

(c) a smooth cubic hypersurface in $\mathbb{P}^{n+1}$;

(d) an intersection of two smooth quadric hypersurfaces in $\mathbb{P}^{n+2}$;

(e) a linear section of the Grassmannian $\mathrm{Gr}(2,5)$ in its plucker embedding.

As a next step, Pym developed the key lemma. We can calculate each variety of high index one by one; we then obtain the conclusion that the variety must be a projective space.

Theorem 3.2 ([14, Prop 5.14]) Let $D=D(\Pi)=\sum_{j=1}^{k} D_{j}$ be a irreducible decomposition of the degeneracy divisor. We write $\left[D_{j}\right]=c_{1}\left(\mathcal{O}_{X}\left(D_{j}\right)\right)$. Then the following 
relation holds in the cohomology rings of $X$ :

$$
\operatorname{ch}\left(\mathcal{T}_{X}\right)-\operatorname{ch}\left(\mathcal{T}_{X}{ }^{\vee}\right)=2 \sum_{j=1}^{k} \sinh \left(\left[D_{j}\right]\right) .
$$

Example 3.3 First case is $X=\mathbb{P}^{2 n}, n \geq 2$. Let $H$ be an ample generator of the Picard group, $D(\Pi)=\sum_{j=1}^{k} D_{j}$ a irreducible decomposition. Then we write $D_{j}=d_{j} H$. The Euler sequence leads the equation $\operatorname{ch}\left(\mathcal{T}_{X}\right)=(2 n+1) e^{H}-1$. Therefore, the left-hand side of (3.1) becomes $2(2 n+1) \sinh [H]$. We compare the intersection number of both sides of (3.1) by intersecting the degree $2 i-1$ cycle $H^{2 i-1}$. Then we obtain

$$
\begin{aligned}
& 2 n+1=\sum_{j=1}^{k} d_{j} \\
& 2 n+1=\sum_{j=1}^{k} d_{j}^{3} \\
& \vdots \\
& 2 n+1=\sum_{j=1}^{k} d_{j}^{2 n-1},
\end{aligned}
$$

where the top equation is obtained when $i=2 n-1$. This straightforward calculation gives a unique solution of integers $d_{j}$ :

$$
\begin{aligned}
d_{j} & =1(1 \leq \forall j \leq k) \\
k & =2 n+1 .
\end{aligned}
$$

Since we suppose that the degeneracy divisor is a simple normal crossing one, it is composed of coordinate hyperplanes.

If the variety is not a projective space, then we can confirm that it is unsuitable in terms of the above lemma. We show this in one case, but it can be confirmed in the remaining cases as well.

Example 3.4 Let $X \subset \mathbb{P}^{2 n+1}$ be a smooth quadric hypersurface. Let $H$ be an ample generator of the Picard group and let $D(\Pi)=\sum_{j=1}^{k} D_{j}$ be an irreducible decomposition. Then we write $D_{j}=d_{j} H$. We have the relative Euler sequence:

$$
0 \longrightarrow \mathcal{T}_{X} \longrightarrow \mathcal{T}_{\mathbb{P}^{2 n+1}} \otimes \mathcal{O}_{X} \longrightarrow \mathcal{N}_{X / \mathbb{P}^{2 n+1}} \longrightarrow 0 .
$$

Therefore, we obtain the chern character

$$
\begin{aligned}
\operatorname{ch}\left(\mathcal{T}_{X}\right) & =\operatorname{ch}\left(\mathcal{T}_{\mathbb{P}^{2 n+1}} \otimes \mathcal{O}_{X}\right)-\operatorname{ch}\left(\mathcal{N}_{X / \mathbb{P}^{2 n+1}}\right) \\
& =\operatorname{ch}\left(\mathcal{T}_{\mathbb{P}^{2 n+1}}\right)-\operatorname{ch}\left(\mathcal{O}_{X}(2)\right)=(2 n+2) e^{H}-1-e^{2 H} .
\end{aligned}
$$

In the similar way, we compare the coefficients

$$
2 n+2-2=\sum_{j=1}^{k} d_{j}
$$




$$
\begin{gathered}
2 n+2-2^{2}=\sum_{j=1}^{k} d_{j}^{3} \\
\vdots \\
2 n+2-2^{2 n-1}=\sum_{j=1}^{k} d_{j}^{2 n-1} .
\end{gathered}
$$

If $n \geq 2$, there are no solutions that satisfy the above system of equations.

Finally, we specify the form of the Poisson structure; r-matrix construction plays an important role.

Definition 3.5 Let $X$ be a variety equipped with an action of Lie group $G$, let $\mathfrak{g}$ be its Lie algebra, and let $r$ be a classical triangular $\mathrm{r}$-matrix for $G$, that is, $[r, r]=0 \in \wedge^{3} \mathfrak{g}$. Then we obtain the Poisson structure on $X$ by pushing $r$ forward along the action map $\mathfrak{g} \rightarrow \Gamma\left(X, \mathcal{T}_{X}\right)$. We call this construction of Poisson structures r-matrix construction.

When $X$ is a $2 n$-dimensional projective space, we have already known that the degree of each irreducible component of the degeneracy divisor is 1 by Example 3.3. Then one can show that such a Poisson structure must be obtained from r-matrix construction for the Lie group $G=\left(\mathbb{C}^{*}\right)^{2 n}$.

\section{Numerical Properties of Fano Products}

Let $X=\prod_{i=1}^{m} X_{i}$ be a variety of dimension $2 n$, let $X_{i}$ be a Fano variety of dimension $n_{i} \geq 3$ and of Picard number 1 with the projection $p_{i}: X \rightarrow X_{i}, \overline{H_{i}}$ an ample generator of $\operatorname{Pic}\left(X_{i}\right)$, and let $H_{i}=p_{i}^{*} \overline{H_{i}}$ be a pull-back of $\overline{H_{i}}$. Recall that the intersection number is obtained by the following formula:

$$
\prod_{i=1}^{m} H_{i}^{d_{i}}= \begin{cases}d>0 & \forall i, d_{i}=n_{i}, \\ 0 & \exists i, d_{i} \neq n_{i},\end{cases}
$$

where a system of integers $d_{i}$ satisfies $\sum_{i=1}^{m} d_{i}=2 n$ and $d$ is a degree of a projective variety $X$.

Proposition 4.1 Settings are the same as the above. We write the irreducible decomposition of the degeneracy divisor $D=D(\Pi)=\sum_{j=1}^{k} D_{j}$. We set $D_{j}=\sum_{i=1}^{m} a_{i j} H_{i}$ for some non-negative integer $a_{i j} \in \mathbb{N}$. Then for every $j$, there uniquely exists $0 \leq i \leq m$ such that $a_{i j} \neq 0$ and $a_{l j}=0$ for all $l \neq i$. This indicates that we obtain

$$
\operatorname{ch}\left(\mathcal{T}_{X_{i}}\right)-\operatorname{ch}\left(\mathcal{T}_{X_{i}}{ }^{\vee}\right)=2 \sum_{j=1}^{k} \sinh \left(\left[a_{i j} \overline{H_{i}}\right]\right) .
$$

Proof Since $X$ is a product of manifolds $X_{i}$, we have $\operatorname{ch}\left(\mathcal{T}_{X}\right)=\sum_{i=1}^{m} p_{i}^{*} \operatorname{ch}\left(\mathcal{T}_{X_{i}}\right)$. By Pym's method, we have

$$
\operatorname{ch}\left(\mathcal{T}_{X}\right)-\operatorname{ch}\left(\mathcal{T}_{X}^{\vee}\right)=2 \sum_{j=1}^{k} \sinh \left(\left[D_{j}\right]\right)=2 \sum_{j=1}^{k} \sinh \left(\sum_{i=1}^{m}\left[a_{i j} H_{i}\right]\right) .
$$


We consider terms of degree 3 of (4.1) and intersection numbers with respect to the $2 n-3-$ cycle $H_{1}^{n_{1}} \cdots H_{i}^{n_{i}-1} \cdots H_{l}^{n_{l}-2} \cdots H_{m}^{n_{m}}$. This detects the coefficient of $H_{i} H_{l}^{2}$ that is not contained in the left side of (4.1). As we calculated the intersection number, we have

$$
0=3 d \sum_{j=1}^{k} a_{i j} a_{l j}{ }^{2} .
$$

This implies that $a_{i j} a_{l j}{ }^{2}=0$ for every $j$. Therefore, for some fixed $j$, if there exists a non-zero integer $a_{i j}$, then we find that the other integers $a_{l j}=0$ for all $l \neq i$. Moreover, since $H_{j}$ is an effective divisor on $X$, such an integer $a_{i j}$ indeed exists. Now, we have $\operatorname{ch}\left(\mathcal{T}_{X}\right)=\sum_{i=1}^{m} \operatorname{ch}\left(\mathcal{T}_{X_{i}}\right)$; thus, the conclusion on the coefficients of $D_{j}$ suggests that the formula drops down to each variety $X_{i}$ and each divisor $\overline{D_{j}}$ on $X_{i}$ such that $p_{i}^{*} \overline{D_{j}}=D_{j}$.

This proposition and the discussion by Pym immediately lead the following corollary.

Corollary 4.2 Settings are the same as in the above proposition. Then $X_{i}=\mathbb{P}^{n_{i}}$ or $i_{X_{i}}<n_{i}-1$, where $i_{X_{i}}$ is the Fano index of $X_{i}$.

\section{Proof of the Main Theorem}

In this section, we complete the proof of the main theorem. The first goal is to show that $X_{i}=\mathbb{P}^{n_{i}}$ for every $i$. For this aim, it is enough to confirm that $i_{X_{i}} \geq n_{i}-1$. In order to prove this, we will construct the triplet $(X, \Pi, D(\Pi))$ inductively. At first, we take any irreducible component of $D(\Pi)$ and name it $D_{1}$. Then it is enough to show that we can choose another component $D_{2}$ such that $\left(Y=D_{1} \cap D_{2},\left.\Pi\right|_{Y}, D\left(\left.\Pi\right|_{Y}\right)=\right.$ $\left.\left.\left(D(\Pi)-D_{1}-D_{2}\right)\right|_{Y}\right)$ is again an SNC log symplectic structure on some Fano product.

\subsection{Lemmas for the Induction}

The settings are the same as in the above section; namely, let $X_{i}$ be a Fano manifold of Picard number 1 and of dimension $n_{i} \geq 3$ over a complex number field $\mathbb{C}$, let $\overline{H_{i}}$ be an ample generator of $\operatorname{Pic}\left(X_{i}\right)$, let $H_{i}=p_{i}^{*} \overline{H_{i}}$ be a pull-back of $\overline{H_{i}}$, let $X=\prod_{i=1}^{m} X_{i}$ be a product of even dimension $2 n=\sum_{i=1}^{m} n_{i}$, let $p_{i}: X \rightarrow X_{i}$ be the $i$-th projection, let $\Pi$ be a SNC log symplectic structure on $X$, let $D=D(\Pi)=\sum_{j=1}^{k} D_{j}$ be the degeneracy divisor and its irreducible decomposition, and let $\overline{D_{j}}$ be a divisor on $X_{i}$ for some $i$ such that $p_{i}^{*} \overline{D_{j}}=D_{j}$.

Lemma 5.1 Let $\Pi$ be a SNC log symplectic structure on $X=\prod_{i=1}^{m} X_{i}$ with $n_{i} \geq 3$ and $\omega=\Pi^{-1}$ a two-form corresponding to $\Pi$, that is, $\Pi \neg \omega=1$. We take an arbitrary irreducible component $D_{j}$ of the degeneracy divisor $D(\Pi)$, and we set the one-form $\alpha=\operatorname{Res}_{D_{j}} \omega$. Then $\alpha$ is not identically zero and has poles along $D_{j} \cap\left(D(\Pi) \backslash D_{j}\right)$.

Proof $\alpha$ is evidently a non-zero meromorphic one-form that may have poles along $D_{j} \cap\left(D(\Pi) \backslash D_{j}\right)$. It is enough to show that $\alpha$ is not holomorphic. We suppose that $\overline{D_{j}}$ is a divisor on $X_{i}$ and set $\bar{X}=\prod_{k \neq i} X_{k}$. Then $D_{j}=\overline{D_{j}} \times \bar{X}$ holds. Applying Kunneth's 
formula, the following equation holds:

$$
H^{0}\left(D_{j}, \Omega_{D_{1}}^{1}\right)=H^{0}\left(\overline{D_{j}}, \Omega_{\overline{D_{j}}}^{1}\right) \otimes H^{0}\left(\bar{X}, \mathcal{O}_{\bar{X}}\right) \oplus H^{0}\left(\overline{D_{j}}, \mathcal{O}_{\overline{D_{j}}}\right) \otimes H^{0}\left(\bar{X}, \Omega_{\bar{X}}^{1}\right) .
$$

By the Kodaira vanishing theorem, the latter term is 0 .

We apply the Lefshetz hyperplane theorem for Hodge decomposition (see e.g., [9, Example 3.1.24]), $X_{i}, \overline{D_{j}}$ and $(p, q)=(0,1)$. Then the equation $H^{0}\left(\overline{D_{j}}, \Omega_{\overline{D_{j}}}\right)=$ $H^{0}\left(X_{i}, \Omega_{X_{i}}^{1}\right)=0$ holds. Therefore, $H^{0}\left(D_{j}, \Omega_{D_{j}}^{1}\right)=0$ and $\alpha$ can not be holomorphic.

Lemma 5.2 (Residue theorem for a product variety) Let $X_{i}$ be a complex manifold with $\operatorname{Pic}\left(X_{i}\right)=\mathbb{Z}\left[H_{i}\right]$ and $H^{1}\left(X_{i}, \mathcal{O}_{X_{i}}\right)=0$, let $X=\prod_{i=1}^{m} X_{i}$ be a product, let $p_{i}: X \rightarrow X_{i}$ be an $i$-th projection, let $D$ be a simple normal crossing divisor on $X$, and let $\alpha \in \Gamma\left(X, \Omega_{X}^{1}(\log D)\right)$ with residues $\lambda_{j}$, that is, $\operatorname{Res}_{D_{j}} \alpha=\lambda_{j}$. We write the irreducible decomposition $D=\sum_{j=1}^{k} D_{j}$ and $D_{j}=\sum_{i} a_{i j} H_{i}$. Then the following equation holds:

$$
\sum_{j=1}^{k} a_{i j} \lambda_{j}=0 \text { for all } i \text {. }
$$

Proof There exists a residue exact sequence

$$
0 \longrightarrow \Omega_{X}^{1} \longrightarrow \Omega_{X}^{1}(\log D) \longrightarrow \oplus_{j} \mathcal{O}_{D_{j}} \longrightarrow 0 .
$$

This induces the long exact sequence

$$
H^{0}\left(X, \Omega_{X}^{1}\right) \longrightarrow H^{0}\left(X, \Omega_{X}^{1}(\log D)\right) \stackrel{\varphi}{\longrightarrow} \oplus_{j} H^{0}\left(D_{j}, \mathcal{O}_{D_{j}}\right) \stackrel{\delta}{\longrightarrow} H^{1}\left(X, \Omega_{X}^{1}\right) .
$$

Now, we have $H^{0}\left(D_{j}, \mathcal{O}_{D_{j}}\right)=H^{0,0}\left(D_{j}\right)=H^{0}\left(D_{j}, \mathbb{C}\right)$. The injection $\iota_{j}: D_{j} \rightarrow X$ induces $\iota_{j}^{*} ; H^{p}(X, \mathbb{C}) \rightarrow H^{p}\left(D_{j}, \mathbb{C}\right)$. Its dual is $\iota_{j}^{* \vee}:\left(H^{p}\left(D_{j}\right)\right)^{\vee} \rightarrow\left(H^{p}(X, \mathbb{C})\right)^{\vee}$. The Poincaré duality suggests that there is a map $\left(H^{p}\left(D_{j}\right)\right)^{\vee}=H^{2 n-2-p}\left(D_{j}, \mathbb{C}\right) \rightarrow$ $H^{2 n-p}(X, \mathbb{C})=\left(H^{p}(X, \mathbb{C})\right)^{\vee}$. In particular, when we take $p=2 n-2$, we obtain the morphism

$$
\iota_{j_{*}}: H^{0}\left(D_{j}, \mathbb{C}\right) \longrightarrow H^{1,1}(X) .
$$

Due to the construction of $\iota_{j_{*}}$, this sends $1 \mapsto\left[c_{1}\left(D_{j}\right)\right]$. Then the connecting morphism $\delta$ is a sum of $\iota_{j_{\star}}$. Recall that we assumed that $H^{1}\left(X_{i}, \mathcal{O}_{X_{i}}\right)=0$, so $\delta$ factors through the inclusion $\operatorname{Pic}(X) \otimes \mathbb{C} \rightarrow H^{1}\left(X, \Omega_{X}^{1}\right)$ and $\operatorname{Pic}(X)=\sum_{i=1}^{m} \operatorname{Pic}\left(X_{i}\right)$. As $\alpha$ is an element of $H^{0}\left(X, \Omega_{X}^{1}(\log D)\right), \delta(\varphi(\alpha))=0$. The $i$-th component contained in $\operatorname{Pic}\left(X_{i}\right)$ of this equation is $\sum_{j=1}^{k} \lambda_{j} a_{i j}=0$.

\subsection{Induction}

Now, we focus on the first component $X_{1}$ and show that $X_{1}=\mathbb{P}^{n_{1}}$. If we can prove this claim, then we can show that $X=\prod_{i=1}^{m} \mathbb{P}^{n_{i}}$ by changing the index of the variety.

We take an irreducible component $D_{1}$ of $D(\Pi)$ and suppose that $\overline{D_{1}}$ is a divisor on $X_{1}$. Then, Lemma 5.1 ensures that there exists some irreducible component $D_{2}$ such that $\operatorname{Res}_{D_{2}}\left(\operatorname{Res}_{D_{1}} \omega\right) \neq 0$.

We will find two cases:
(A) $\overline{D_{2}}$ is a divisor on $X_{1}$,
(B) $\overline{D_{2}}$ is a divisor on $X_{2}$. 
Case A. We set $Y_{1}=\overline{D_{1}} \cap \overline{D_{2}}, D_{Y}=\left(D(\Pi)-D_{1}-D_{2}\right) \cap Y$, and $\Pi_{Y}=\left.\Pi\right|_{Y}$. Then [14, Lemma 5.18] ensures that $\Pi_{Y}$ is a generically symplectic Poisson structure. By construction, we can write $Y=Y_{1} \times \prod_{i=2}^{m} X_{i}$. Applying the residue theorem for $\alpha=\operatorname{Res}_{D_{1}} \omega$ and the variety $D_{1}=\overline{D_{1}} \times \prod_{i=2}^{m} X_{i}$, we obtain the third irreducible component $D_{3}$ such that $\overline{D_{3}}$ is a divisor on $X_{1}$. Then $D_{3}$ ensures that $D_{Y}$ is non-empty, and so $D_{Y}$ is a simple normal crossing divisor. The adjunction formula indicates that $D_{Y}$ is an anti-canonical divisor; that is, $Y_{i}$ is a Fano variety. Owing to the construction of $Y$, we have $D\left(\left.\Pi\right|_{Y}\right)=D_{Y}$. If $n_{1} \geq 5$, by applying the Lefshetz hyperplane theorem twice, we see that $H^{2}\left(X_{1}, \mathbb{Z}\right) \rightarrow H^{2}\left(Y_{1}, \mathbb{Z}\right)$ is an isomorphism. Thus, $Y_{i}$ is also the variety of Picard number 1 . Therefore, we obtain a new SNC log symplectic triplet $\left(Y=\prod_{k \neq i} X_{k} \times Y_{i}, \Pi_{Y}\right)$ with $\operatorname{dim} Y_{1}=n_{1}-2$.

Next, we consider the case where $n_{1}=3,4$. Then $Y_{i}$ does not satisfy the hypothesis of Lefshetz hyperplane theorem. As we have three divisors $\overline{D_{1}}, \overline{D_{2}}, \overline{D_{3}}$ on $X_{i}$, the Fano index $i_{X_{1}}$ of $X_{1}$ becomes greater than 3 . Since we suppose that $n_{1} \leq 4$, Corollary 4.2 indicates that $X_{1}=\mathbb{P}^{n_{1}}$. Moreover, as we computed in Example 3.3, we see that the degree $a_{i j} \neq 0$ of the divisor $D_{j}$ equals 1 . This means that we just obtain the projective space as a degree 1 hyperplane of the projective space when we cut out some irreducible component $D_{1}, D_{2}$ of $D(\Pi)$ in the step of the induction. Therefore, we can discuss this in the same way, because of the two facts, that is, $H^{0}\left(\mathbb{P}^{n}, \Omega_{\mathbb{P}^{n}}^{1}\right)=0$ and Picard number of the projective space is 1 . Finally, we can also replace $X$ with $Y$ even if $n_{1}=3,4$.

Case B. Now, we have $Y=D_{1} \cap D_{2}=\overline{D_{1}} \times \overline{D_{2}} \times \prod_{i=3}^{m} X_{i}, Y_{1}=\overline{D_{1}}$. We can also find that $D\left(\left.\Pi\right|_{Y}\right)=\left.\left(D(\Pi)-D_{1}-D_{2}\right)\right|_{Y}$. Then the residue theorem ensures that there exist $D_{4}$ such that $\overline{D_{4}}$ is a divisor on $X_{2}$ in the similar way as above. By switching the role of $D_{1}$ and $D_{2}$, we obtain $D_{3}$ with $\overline{D_{3}}$ is a divisor on $X_{1}$. Therefore, $\overline{D_{1}}$ and $\overline{D_{2}}$ is a Fano variety. If $n_{1} \geq 4$ and $n_{2} \geq 4$, then we can apply the Lefshetz hyperplane theorem and obtain that $\rho\left(\overline{D_{1}}\right)=\rho\left(\overline{D_{2}}\right)=1$.

Next, we suppose $n_{i}=3$, for $i=1$ or 2 . The Fano index $i_{X_{i}}$ of $X_{i}$ is greater than 2 , since $D(\Pi)$ has at least 2 components $D_{i}$ and $D_{i+2}$ that are derived from $X_{i}$. Therefore, Corollary 4.2 leads to $X_{i}=\mathbb{P}^{3}$.

Dimension $\leq \mathbf{2}$ and Summary. The outline of our inductive process is as follows. First, we take $D_{1}=a_{11} p_{1}^{*} \overline{H_{1}}$. Next, we take $D_{2}$ whose existence is ensured by Lemma 5.1. Although we distinguish two cases by the source of $D_{2}$, in any case, we obtain the SNC log symplectic triplet $\left(Y,\left.\Pi\right|_{Y}, D_{Y}\right)$. Then we take $D_{1}$ again and repeat steps until we find that $Y_{1}$ is a projective space, and this will be achieved when the dimension becomes smaller than 3 . Finally, we go back through the induction steps one by one and count the Fano index; then we find that $X_{1}=\mathbb{P}^{n_{1}}$.

Before the conclusion, we note the following. On the above construction, we treat the case of $n_{i} \geq 3$, that is, our initial condition. In both Case $\mathrm{A}$ and Case $\mathrm{B}$, the step reduces the dimension of the component. In particular, when we repeat Case B, $n_{2}=\operatorname{dim} X_{2}$ may be equal to or smaller than 2, and this is out of our assumptions. But if we encounter a such case, then that component must pass through Case B of $n_{2}=3$. This means that we can treat such a low-dimensional component as a degree 1 
hyperplane cut of the projective space. Thus, our induction works for every stage of the inductive process.

\subsection{Poisson Structures on the Product of Projective Spaces}

Proposition $5.3 \quad\left[14\right.$, Exercise 5.17] Let $\left(X=\prod_{i=1}^{m} \mathbb{P}^{n_{i}}, \Pi\right)$ be a SNC log symplectic structure with $n_{i} \geq 3$. Then, $\Pi$ must be induced by r-matrix construction for the standard action of the torus $G=\left(\mathbb{C}^{*}\right)^{n_{1}} \times\left(\mathbb{C}^{*}\right)^{n_{2}} \times \cdots \times\left(\mathbb{C}^{*}\right)^{n_{m}}$.

Proof Let $Y$ be an orbit of a general point $x$ and let $\mathfrak{g}$ be a Lie algebra of $G$. The action of $G$ preserves the degeneracy divisor $D(\Pi)=X \backslash Y$. For the action map $a: \mathfrak{g} \rightarrow$ $\Gamma\left(Y, \mathcal{T}_{Y}\right)$ and the element $\xi \in \mathfrak{g}$, we construct an element $\xi_{X} \in \Gamma\left(X, \mathcal{T}_{X}\right) . \xi_{X}$ satisfies two conditions, $\left.\xi_{X}\right|_{Y}=a(\xi)$ and $\left.\xi_{X}\right|_{U}=0$ for any open subset $U$ contained in $X \backslash Y$. So we obtain the map $\widetilde{a}: \mathfrak{g} \rightarrow \Gamma\left(X, \mathcal{T}_{X}\right)$.

Next, we consider the basis of $\Gamma\left(X, \mathcal{T}_{X}(-\log D)\right)$. For a standard affine coordinate $x_{1}, \ldots, x_{n}$, the vector fields $x_{1} \partial x_{1}, \ldots, x_{n} \partial x_{n}$ form a basis on that affine open set. As they can glue to that on a product of projective spaces, we see that they form a global basis for $\mathcal{T}_{X}(-\log D)$. Therefore, $\mathcal{T}_{X}(-\log D)$ is a trivial bundle over $X$ whose fibers are canonically identified with $\mathfrak{g}$. Since holomorphic sections of a trivial bundle on a compact manifold are always constant, we obtain the isomorphism $\wedge^{2} \mathfrak{g} \simeq \Gamma\left(X, \wedge^{2} \mathcal{T}_{X}(-\log D)\right)$ as a vector space.

When we take coordinates $\left(\left[x_{10}: x_{11}: \cdots: x_{1 n_{1}}\right],\left[x_{20}: \cdots: x_{2 n_{2}}\right], \ldots,\left[x_{m 0}:\right.\right.$ $\left.\left.\cdots: x_{m n_{m}}\right]\right)$, this means that Poisson structure $\Pi$ is an invariant against the following transformation:

$$
\begin{aligned}
& x_{i 0} \longmapsto x_{i 0} \\
& x_{i j} \longmapsto c_{i j} x_{i j},
\end{aligned}
$$

where $j \neq 0$ and $c_{i j} \in \mathbb{C}^{*}$. We can write the Poisson structure by using the coordinate as follows:

$$
\Pi=\sum_{i j>k l, 0 \leq j \leq n_{i}, 0 \leq l \leq n_{k}}\left\{x_{i j}, x_{k l}\right\} \frac{\partial}{\partial x_{i j}} \wedge \frac{\partial}{\partial x_{k l}} .
$$

When we set $y_{i j}=c_{i j} x_{i j}$, we have $\frac{\partial}{\partial y_{i j}}=\frac{\partial x_{i j}}{\partial y_{i j}} \frac{\partial}{\partial x_{i j}}=\frac{1}{c_{i j}} \frac{\partial}{\partial x_{i j}}$. Therefore, $\left\{x_{i j}, x_{k l}\right\}$ must be divisible by $x_{i j}$. For the same reason, $x_{k l}$ also divides by $\left\{x_{i j}, x_{k l}\right\}$. Therefore, the following equation holds:

$$
\left\{x_{i j}, x_{k l}\right\}=c_{i j k l} x_{i j} x_{k l}
$$

for some coefficient $c_{i j k l} \in \mathbb{C}$. For the general choice of the coefficients $c_{i j k l}$, in order to make $\Pi$ be a generically symplectic, the degeneracy divisor is a union of all coordinate hyperplanes. One can also confirm that bivector fields of this form vanishes the Schouten bracket.

Acknowledgments The author would like to express his gratitude to his supervisor professor Yasunari Nagai for a lot of discussions and encouragement. He also thanks Professor Brent Pym for beneficial comments on the Residue Theorem applied in his research [14]. He is also grateful to Professor Hajime Kaji for helpful comments. 
The author has had the support and encouragement of Daizo Ishikawa, Ryo Okawa, and Taku Suzuki.

\section{References}

[1] G. R. Cavalcanti, Examples and counter-examples of log-symplectic manifolds. J. Topol. 10(2017), 1-21. https://doi.org/10.1112/topo. 12000

[2] T. Fujita, Classifications of projective varieties of $\Delta$-genus one. Proc. Japan Acad. Ser. A Math. Soc. 58(1982), 113-116.

[3] T. Fujita, On polarized varieties of small $\Delta$-genera. Tohoku Math. J. (2) 34(1982), 319-341. https://doi.org/10.2748/tmj/1178229197

[4] R. Goto, Rozansky-witten invariants of log symplectic manifolds. In: Integrable systems, topology, and physics (Tokyo, 2000). Comtemp. Math., 309, Amer. Math. Soc., Providence, RI, 2002, pp. 69-84. https://doi.org/10.1090/conm/309/05342

[5] M. Gualtieri and S. Li, Symplectic groupoids of log symplectic manifolds. Int. Math. Res. Not. IMRN 2014, no. 11, 3022-3074. https://doi.org/10.1093/imrn/rnt024

[6] M. Gualtieri and B. Pym, Poisson modules and degeneracy loci. Proc. Lond. Math. Soc. 107(2013), 627-654. https://doi.org/10.1112/plms/pds090

[7] V. Guillemin, E. Miranda, and A. R. Pires, Symplectic and poisson geometry on b-manifolds. Adv. Math. 264(2014), 864-896. https://doi.org/10.1016/j.aim.2014.07.032

[8] S. Kobayashi and T. Ochiai, Characterizations of complex projective spaces and hyperquadrics. J. Math. Kyoto Univ. 13(1973), 31-47. https://doi.org/10.1215/kjm/1250523432

[9] R. Lazarsfeld, Positivity in algebraic geometry. Vol. 1, Springer-Verlag, Berlin, 2004. https://doi.org/10.1007/978-3-642-18808-4

[10] R. Lima and J. V. Pereira, A characterization of diagonal poisson structure. Bull. Lond. Math. Soc. 46(2014), 1203-1217. https://doi.org/10.1112/blms/bdu074

[11] I. Măcut and B. O. Torres, Deformations of log-symplectic structures. J. Lond. Math. Soc. (2) 90(2014), 197-212. https://doi.org/10.1112/jlms/jdu023

[12] K. G. O'Grady, A new six-dimensional irreducible symplectic variety. J. Algebraic Geom. 12(2003), 435-505. https://doi.org/10.1090/S1056-3911-03-00323-0

[13] K. Okumura, A classification of SNC log symplectic structures on blow-up of projective spaces.

[14] B. Pym, Constructions and classifications of projective poisson varieties. Lett. Math. Phys. 108(2017), 573-632. https://doi.org/10.1007/s11005-017-0984-5

[15] B. Pym, Elliptic singularities on log symplectic manifolds and Feiginn-Odesskii Poisson brackets. Compos. Math. 153(2017), 717-744. https://doi.org/10.1112/S0010437X16008174

[16] O. Radko, A classification of topologically stable poisson structures on a compact oriented surface. J. Symplectic Geom. 1(2002), 523-542.

Department of Mathematics, Waseda University, Ookubo, Shinjuku-ku, Tokyo, 169-8555, Japan

e-mail: k_okumura@moegi.waseda.jp 